\title{
PROBING NON-AXISYMMETRY WITH PROPER MOTIONS
}

\author{
JAMES BINNEY \\ Department of Physics \\ 1 Keble Road, Oxford, OX1 3NP, England
}

\begin{abstract}
The potential of the Milky Way is almost certainly not axisymmetric: the centre is believed to be dominated by a bar, and beyond the solar radius some non-axisymmetric feature of the potential appears to warp the disk. There are grounds for believing that the outer potential is mildly elliptical. Sub mas $\mathrm{yr}^{-1}$ proper motions of objects that lie near the plane several kiloparsecs from the Sun would play a crucial role in refining our understanding of these non-axisymmetries.
\end{abstract}

\section{Introduction}

Until recently it has usually been assumed that the Milky Way is fundamentally an axisymmetric system - the only non-axisymmetric features in its gravitational potential were assumed to arise from spiral structure. In the last few years two potential sources of more significant non-axisymmetries have attracted attention: (i) triaxiality of the Milky Way's dark halo would be expected to distort the outer galaxy from axisymmetry, and (ii) several independent arguments point towards the inner $\sim 2 \mathrm{kpc}$ of the Milky Way being dominated by a rapidly rotating bar. My purpose here is to ask what high-precision astrometry can contribute to our knowledge of the shape of the Milky Way.

At optical wavelengths obscuration severely limits our ability to probe the large-scale structure of the Milky Way. Since the severity of the problem posed by obscuration has steadily lessened as technological advances have increased the sensitivity of detectors and opened up new wavebands, I shall ignore obscuration in this exploratory survey. Thus I ask 'what could be learned about non-axisymmetry in the Milky from proper motions?' without regard to the feasibility of obtaining the necessary data. 
My treatment will be superficial in that I shall largely ignore the interconnectedness of most measurements of galactic structure. The great variety of the observational material available to us--the position, parallax, proper motion, radial velocity, spectral type and metallicity of a star are in principle all measurable - can be adequately exploited only by comparing them with pseudo-observations of dynamical models of the Milky Way (e.g., Binney 1994). Since such models are not yet available, we cannot yet fully exploit kinematical observations.

\section{NON-AXISYMMETRY OF THE OUTER GALAXY}

As is now well known, the circular-speed curve of the Milky Way is flat or continues to rise at the solar radius $R_{0}$ and beyond (e.g., Fich \& Tremaine 1991), whereas the prediction of models in which the disk, bulge and metalpoor halo all have position-independent mass-to-light ratios $\Upsilon$, is that the circular speed $v_{c}$ should decline from about $R_{0}$ outwards. This situation could simply indicate that $\Upsilon_{\text {disk }}$ increases outwards. Indeed, the detection of gravitational micro-lensing events along lines of sight to the Magellanic Clouds and the bulge offers some support to this conjecture by suggesting the existence of dynamically important numbers of sub-stellar objects. If the flatness of the rotation curve is caused by an increase with radius of $\Upsilon_{\text {disk }}$, it would be natural for the outer galaxy to be axisymmetric. However, it is widely suspected that $v_{c}$ is held constant by a dark halo of nonbaryonic matter. A non-baryonic dark halo would be dynamically distinct from the disk, and there are reasons for believing that it would be nonaxisymmetric (Binney 1978, Frenk et al. 1988, Warren et al. 1992), and/or that its principal axes would not coincide with those of the Milky Way's visible components (Dekel \& Shlosman 1983, Toomre 1983).

\subsection{KINEMATIC EFFECTS OF TRIAXIALITY}

Kuijken \& Tremaine (1994) have presented a detailed investigation of the kinematic effects of a triaxial halo, albeit with emphasis on the role of radial velocities rather than proper motions. They suppose that the intersection of the Milky Way's equi-potential surfaces with the plane have ellipticity $\epsilon_{\Phi}$, and that the short axes of these ellipses make angle $\phi_{b}$ with the Suncentre line. Then they seek constraints on the quantities $c_{\Phi} \equiv \epsilon_{\Phi} \cos 2 \phi_{b}$ and $s_{\Phi} \equiv \epsilon_{\Phi} \sin 2 \phi_{b}$.

Three local measures lead to an interesting upper limit, $\left|s_{\Phi}\right| \lesssim 0.05$, on $s_{\Phi}$. These are (i) the value of the vertex deviation $l_{v}$ (the angle between the long axis of the local velocity ellipsoid and the direction to the galactic centre) for stars of velocity dispersion $\sigma_{r} \gtrsim 30 \mathrm{~km} \mathrm{~s}^{-1}$ (which may be assumed to be phase-mixed and little affected by very local features such as 


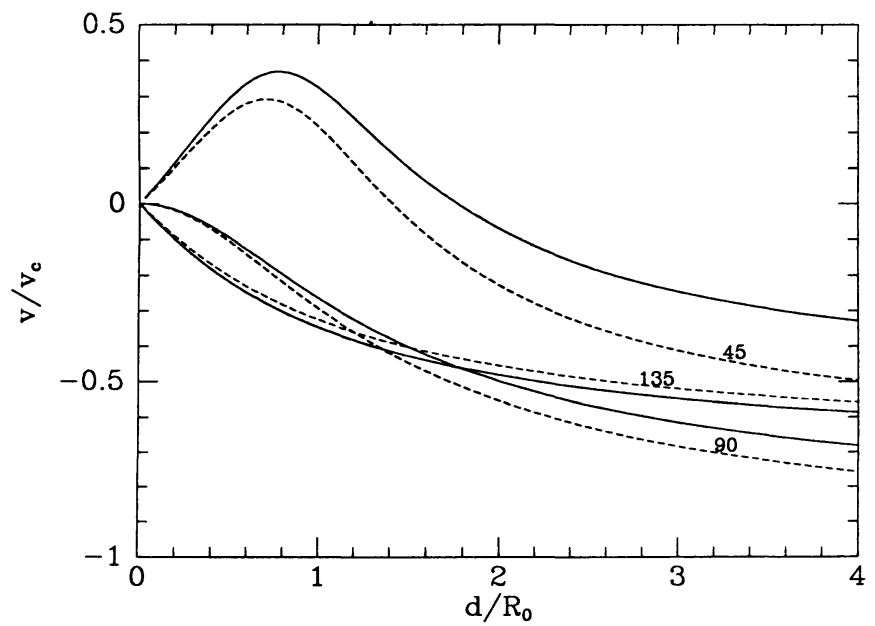

Figure 1. Line-of-sight velocities for material seen at distance $d$ down three lines of sight $\left(l=45^{\circ}, 90^{\circ}, 135^{\circ}\right)$ in a galaxy with flat circular-speed curve and elogated potential ( $s_{\Phi}=0, c_{\Phi}=0.1$ and in the corresponding axisymmetric galaxy (full and dashed curves).

spiral arms), and (ii) the values of the constants $K$ and $C$ that are defined by the generalized Oort equations for the line-of-sight velocity and proper motion of a disk object seen at distance $d$ at galactic longitude $l$,

$$
\begin{aligned}
v_{\mathrm{los}}(d, l) & =(K+A \sin 2 l+C \cos 2 l) d+\mathrm{O}\left(d^{2}\right) \\
\mu(d, l) & =B+A \cos 2 l-C \sin 2 l+\mathrm{O}(d) .
\end{aligned}
$$

The values of $K, C$ and $l_{v}$ are all consistent with zero. This conclusion is reinforced by the limit $\left|v_{R}(\mathrm{LSR})\right|<9 \mathrm{~km} \mathrm{~s}^{-1}$ placed on the radial component of velocity of the LSR by observations of objects such as globular clusters and $\mathrm{OH} / \mathrm{IR}$ stars that should have no net motion with respect to the centre. For $\epsilon_{\Phi}=0.1$, the cited upper limit on $s_{\Phi}$ implies that the Sun lies within $\sim 15^{\circ}$ of a principal axis.

Given that we lie close to a principal axis, it is difficult measure $\epsilon_{\Phi}$ from radial velocities alone. In Figure 1 radial velocity $v$ is plotted as a function of distance $d$ along lines of fixed $l$ for the cases $c_{\Phi}=0.1$ (full curves) and $c_{\Phi}=0$ (dashed curves). Although $c_{\Phi}$ significantly affects the radial velocities observed at given $(d, l)$, the shapes of the full and dashed curves are similar, so the value of $c_{\Phi}$ cannot be determined unless one somehow knows the underlying circular-speed curve, or knows the distances to observed objects in terms of $R_{0}$. Figure 2, which plots the ratio $d_{v} \equiv v_{\text {los }} / \mu_{l}$ for the models and lines of sight of Figure 1, suggests how accurate proper motions could be used to resolve this ambiguity. This ratio is clearly independent of the velocity normalization of the model, and one cannot simultaneously 


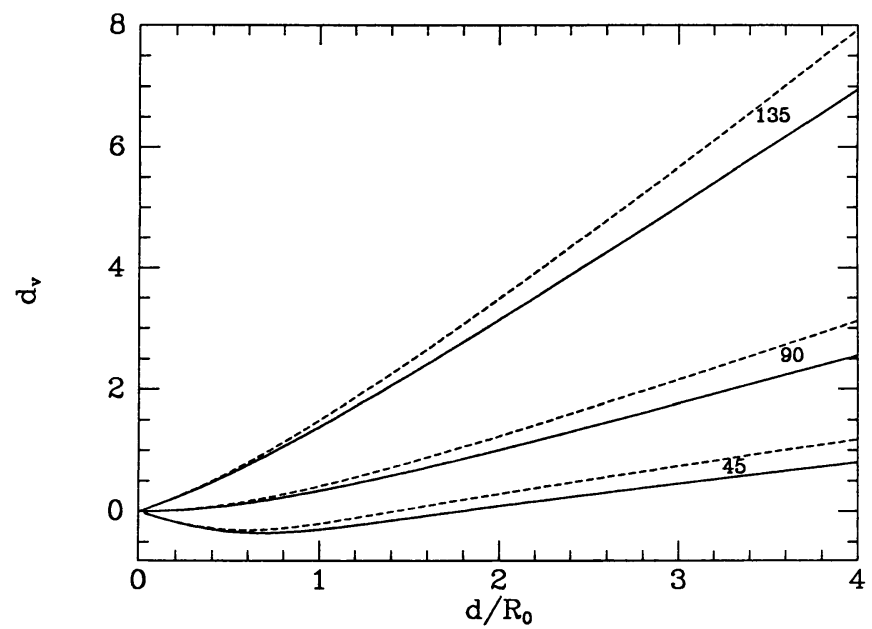

Figure 2. The ratio $d_{v} \equiv v_{\mathrm{los}} / \mu_{l}$ for objects seen at distance $d$ down three lines of sight $\left(l=45^{\circ}, 90^{\circ}, 135^{\circ}\right)$ in a galaxy with elogated potential $\left(s_{\Phi}=0, c_{\Phi}=0.1\right.$; full curves $)$ and in the corresponding axisymmetric galaxy (dashed curves).

superimpose all three full curves on the corresponding dashed curves by a single revision of $R_{0}$. For example, the curves for $l=135^{\circ}$ require that $R_{0}$ be revised by a smaller factor than do the curves for $l=45^{\circ}$. Hence if one could make this plot from accurate observational material, one could determine whether the outer Milky Way is axisymmetric or not without knowledge of either $v_{c}$ or $R_{0}$.

Although Kuijken \& Tremaine conclude that $c_{\Phi}$ cannot be determined by radial velocities alone, they obtain an estimate, $c_{\Phi} \simeq 0.08$ from Merrifield's (1992) discussion of the flaring of the HI disk. Kuijken \& Tremaine point out that this value is supported by the local value of the Oort ratio $X^{2} \equiv \sigma_{\phi}^{2} / \sigma_{R}^{2}=0.42 \pm 0.06$. This value is considerably smaller than that expected for a flat or rising circular-speed curve (e.g. Cuddeford \& Binney 1994), but is compatible with $v_{c}$ constant at $v_{c}=197 \pm 9 \mathrm{~km} \mathrm{~s}^{-1}$ and $c_{\Phi}=0.08 \pm 0.014$. In the model favoured by Kuijken \& Tremaine, the Sun's instantaneous angular velocity is $\Omega_{0}=5.1 \pm 0.6 \mathrm{mas} \mathrm{yr}^{-1}$. If one makes the plausible assumption that the peculiar motion of the massive black hole candidate Sgr $\mathrm{A}^{*}$ is negligible, then the VLBI proper-motion study of Backer (1994) amounts to a direct measurement of $\Omega_{0}=6.55 \pm 0.34 \mathrm{masyr}^{-1}$ in mild conflict with the prediction of Kuijken \& Tremaine.

\subsection{KINEMATIC SIGNATURE OF A WARP}

Whether or not the dark halo is triaxial, its principal axes are likely to be misaligned with those of the inner galaxy. Such misalignment would provide 
a natural explanation of the fact that the disk of the Milky Way, like many other galactic disks is warped (e.g. Binney 1992). The shape of the disk, which is flat inside $r \simeq 10 \mathrm{kpc}$ and then bends to the north at $l>0$ and southwards at $l<0$ is most easily traced in the distribution of the Milky Way's gas (Henderson, Jackson \& Kerr 1982). Recently the DIRBE/COBE team has shown that the stellar disk is also warped (Freudenreich et al. 1994) as would be expected if the warp in the gas layer arises from misalignment of the potentials of the dark halo and inner galaxy.

In a popular theory of how this misalignment results in a warp (Sparke \& Casertano 1988), the entire warped disk is precessing about the symmetry axis of the dark halo as if it were a rigid body. Let $\omega_{p}$ be the angular velocity of the precession. Then the velocity of a star at position $\mathbf{r}$ is $\mathbf{v}=$ $\left(\Omega(r)+\omega_{p}\right) \times \mathbf{r}$, where $\Omega$ is the local circular frequency. Extracting the perpendicular component of this by dotting through with the unit vector $-\widehat{\Omega}\left(r_{\odot}\right)\left[\Omega\left(r_{\odot}\right)\right.$ points to the South], we find

$$
\mu_{b}(\mathbf{r})=\frac{\left(\mathbf{r}-\mathbf{r}_{\odot}\right) \cdot \omega_{p} \times \hat{\Omega}\left(r_{\odot}\right)+\boldsymbol{\Omega}(r) \times \hat{\Omega}\left(r_{\odot}\right) \cdot \mathbf{r}}{\left|\mathbf{r}-\mathbf{r}_{\odot}\right|}
$$

$\boldsymbol{\omega}_{p}$ is perpendicular to the halo's equatorial plane and thus perpendicular to the warp's line of nodes (which is the intersection of the equatorial planes of halo and inner disk). At large radii the disk is expected to align with the halo's plane. So a lower limit on the angle $\psi$ between $-\boldsymbol{\Omega}\left(r_{\odot}\right)$ and $\boldsymbol{\omega}_{p}$ is given by the tilts of the largest reliably observed rings; since the ring at $r=20 \mathrm{kpc}$ rises $\sim 3 \mathrm{kpc}$ out of the plane of the inner disk, we have $\sin \psi \simeq \tan \psi \gtrsim \frac{3}{20}$. Finally exploiting the fact that the Sun lies near the line of nodes, we have for objects in the flat inner disk at $l=0$ or $l=180^{\circ}$

$$
\mu_{b} \simeq \pm \frac{3}{20} \omega_{p}
$$

where the plus sign applies at $l=0$ and minus sign at $l=180^{\circ}$. The second, less informative, term in (3) is non-zero for objects at $r \gtrsim 10 \mathrm{kpc}$. From Figs $1 \& 2$ of Sparke \& Casertano (1988) one finds $\omega_{p} \simeq 0.014\left(v_{c} / r_{d}\right)\left(\epsilon_{\Phi} / 0.07\right)$. So adopting $r_{\odot} / r_{d}=2$ and $\Omega\left(r_{\odot}\right)=6.5 \mathrm{masyr}^{-1}$, we have finally

$$
\mu_{b}(l=0, r \lesssim 10 \mathrm{kpc}) \simeq 0.027\left(\epsilon_{\Phi} / 0.07\right) \operatorname{masyr}^{-1} .
$$

If $\operatorname{Sgr} \mathrm{A}^{*}$ is indeed a massive black hole, its peculiar motion might be small enough for its proper motion to be given by (5). The uncertainty in $\mu_{b}\left(\operatorname{Sgr~A}{ }^{*}\right)$ is currently $\sim 0.23$ mas yr $^{-1}$ (Backer 1994). Alternatively, equation (5) could be checked by averaging the values of $\mu_{b}$ for all stars in the centre and anticentre directions. 


\section{Triaxiality of the bulge}

Several independent lines of evidence now point to the inner $\sim 2 \mathrm{kpc}$ of the Milky Way being dominated by a bar, which is probably rapidly rotating (e.g. Weiland et al. 1994; Stanek et al. 1994). The nearer end of the bar is thought to lie at positive longitudes, such that the line from the centre to the Sun makes an angle of $15^{\circ}$ to $45^{\circ}$ with the long axis of the bar. It has been argued that the distribution and motions of interstellar gas clouds suggest that corotation lies near $R_{\text {corot }}=2.5 \mathrm{kpc}$ (Binney et al. 1991; hereafter BGSBU) although larger values of $R_{\text {corot }}$ are also popular (e.g. Combes 1994).

Spaenhauer, Jones \& Whitford (1992) have determined the proper motions of $429 \mathrm{~K}$ and $\mathrm{M}$ giants in Baade's window $\left(l=1^{\circ}, b=-4^{\circ}\right)$. Unfortunately, these are relative proper motions and it remains to determine the mean motion of the sample. However, Zhao Rich \& Spergel (1994) find evidence for the bar in the kinematics of the 62 stars of the Spaenhauer et al. sample for which Rich $(1988,1990)$ has obtained spectra. Zhao et al. divide these stars into metal-poor $([\mathrm{Fe} / \mathrm{H}]<-0.2)$ and metal-rich $([\mathrm{Fe} / \mathrm{H}]>0)$ groups, containing 15 and 39 stars, respectively. By assuming that all the stars lie $8 \mathrm{kpc}$ from the Sun and using Rich's radial velocities, Zhao et al. plot the space velocities of the two groups. If the galactic potential were axisymmetric, one would expect the projections onto the $\left(v_{r}, v_{l}\right)$ plane of the groups' velocity ellipsoids to align with the $\left(v_{r}, v_{l}\right)$ directions. Actually, neither ellipse aligns with these directions, and the major axes of the two ellipses are nearly at right angles to one another.

Zhao et al. analyse this phenomenon in terms of orbits in a threedimensional generalization of the BGSBU bar model. They find that most of the orbits that carry stars through Baade's window are stochastic rather than regular, and can be broadly classified into prograde and retrograde. The ellipse in the $\left(v_{r}, v_{l}\right)$ plane formed by the velocities of a suitable selection of prograde orbits has an orientation similar to that of the metal-rich stars, while the velocity ellipse of a selection of retrograde orbits resembles the ellipse of the metal-poor stars. Unfortunately, it is not possible to determine the sense of rotation of observed stars without very much more accurate distances to these stars than are currently available.

The $n$-body model of the galactic bulge presented by Sellwood (1993) suggest that the Zhao et al. result has a deep evolutionary significance. Since the earliest days of $n$-body simulations of galactic disks, it has been known that bars form naturally in such systems. More recently it has become clear that bars formed in thin disks can have appreciable vertical extension (Combes et al. 1990, Raha et al. 1991). Moreover, when seen in projection from a point in the original disk plane, these thickened bars 

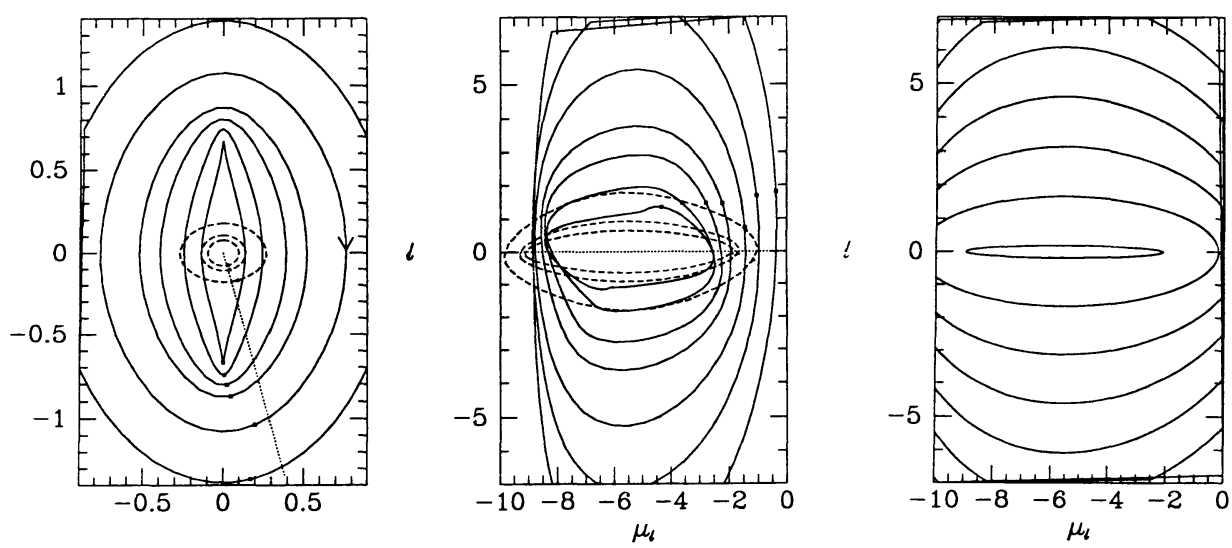

Figure 3. Orbits in the BGSBU model of the bulge/bar. The left-hand panel shows the orbits in real space with the axes marked in kpc; the diagonal straight line points towards the Sun's position. The centre panel shows as a function of $l$ the proper motions $\mu_{l}$ of particles on these orbits in mas $\mathrm{yr}^{-1}$. The right-hand panel shows how the centre panel would look if the potential had the same circular speed curve $v_{c}(r)$ but were axisymmetric. Stars circulate clockwise in the left-hand panel and anti-clockwise in the other two panels. In the left panel $x_{1}$ orbits are shown by full curves and $x_{2}$ orbits by dashed curves.

have the peanut-like shape characteristic of the bulges of some edge-on disk galaxies, including our own. Zhao et al. find that the ellipsoid in the $\left(v_{r}, v_{l}\right)$ plane formed by the velocities of stars at $(l, b)=\left(1^{\circ},-4^{\circ}\right)$ in Sellwood's model, is similar to the ellipse of the metal-rich stars. This suggests that the metal-rich stars formed in the thin disk, and that only the metal-poor stars are part of the old, slowly rotating, bulge.

Observations in the infrared and radio bands make it possible to do astrometry of the bulge outside Baade's window (e.g. Blum, Sellgren \& Terndrup 1994), which, from the dynamical point of view, is inconveniently close to the galactic rotation axis. Figure 3 shows where objects such as gas clouds and young stars, that move on nearly closed orbits, would be expected to lie in the $\left(l, \mu_{l}\right)$ plane. The left-hand panel shows several closed orbits in real space. The centre panel shows the orbits' traces in the $\left(l, \mu_{l}\right)$ plane when viewed along the dotted line of sight of the left panel. Since the assumed velocity of the Sun $\left(220 \mathrm{~km} \mathrm{~s}^{-1}\right)$ is larger than the peak tangential velocity of any of these orbits, the $\left(l, \mu_{l}\right)$ traces are dominated by the reflex of the Sun's motion, and are confined by it to $\mu_{l}<0$. For comparison, the right-hand panel shows the $\left(l, \mu_{l}\right)$ traces of circular orbits in a potential with the same underlying circular-speed curve as the BGSBU model. The nonaxisymmetry of the BGSBU potential has the effect of restricting the range in $\mu_{l}$ associated with the $x_{1}$ orbits. This effect is particularly pronounced at negative $\mu_{l}$, where the model predicts that there should be a pronounced accumulation of objects around $\mu=-8.5$ mas yr$^{-1}$ for $|l| \lesssim 4^{\circ}$. 


\section{Conclusions}

If proper motions of sub mas $\mathrm{yr}^{-1}$ accuracy can be secured for objects that are widely distributed through the Milky Way, we should be able to constrain strongly suspected asymmetries due both to the dark halo and the central bar. Clearly the requirement for a wide distribution of objects with proper motions will be hard to meet at optical wavebands. Moreover, since the predicted motions are small and common to nearly all objects in a given field, it is essential that proper motions be relative to the frame established by extragalactic objects.

\section{References}

Backer, D.C., 1994. In Unsolved Problems of the Milky Way, ed. Blitz, L., Kluwer, Dordrecht

Binney, J.J., 1978. MNRAS, 183, 779

Binney, J.J., 1992. ARA\&A, 30, 51

Binney, J.J., 1994. In Galactic and solar system optical astrometry, ed. L. Morrison, p. 141, Cambridge University Press, Cambridge

Binney, J.J., Gerhard, O.E., Stark, A.A, Bally, J, Uchida, K.I., 1991. MNRAS, 252, 210

Blum, R.D., Sellgren, K, Terndrup, D.M., 1994. In Unsolved Problems of the Milky Way, ed. Blitz, L., Kluwer, Dordrecht

Combes, F., 1994. In Unsolved Problems of the Milky Way, ed. Blitz, L., Kluwer, Dordrecht

Combes, F., Debbasch, F., Friedli, D., Pfenniger, D., 1990. A\&A, 233, 82

Cuddeford, P., Binney, J.J., 1994. MNRAS, 266, 273

Dekel, A., Shlosman, I., 1983. In IAU Symposium 100, Internal kinematics 63 dynamics of galaxies ed. Athanassoula, E., pp. 187-188, Reidel, Dordrecht

Fich, M., Tremaine, S., 1991. ARA\&A, 29, 409

Frenk, C.S., White, S.D.M., Davis, M., Efstathiou, G., 1988. ApJ, 327, 507

Freudenreich, H.T., et al., 1994. ApJ, 429, L69

Kuijken, K., Tremaine, S.D., 1994. ApJ, 421, 178

Henderson, A.P., Jackson, P.D., Kerr, F.J., 1982. ApJ, 263, 116

Merrifield, M.R., 1992. AJ, 103, 1552

Raha, N., Sellwood, J.A., James, R.A., Kahn, F.D., 1991. Nat, 352, 411

Rich, R.M., 1988. AJ, 95, 828

Rich, R.M., 1990. ApJ, 362, 604

Sellwood, J.A., 1993. In Back to the Galaxy, eds Holt, S.S., Verter, F., p. 133, AIP, New York

Spaenhauer, A., Jones, B.F., Whitford, A.E., 1992. AJ, 103, 297

Sparke, L., Casertano, S., 1988. MNRAS, 234, 873

Stanek, K.Z., Mateo, M., Udalski, A., Szymanski, M., Kaluzny, J., Kubiak, M., 1994. ApJ, 429, L73

Toomre, A., 1983. In IAU Symposium 100, Internal kinematics \& dynamics of galaxies ed. Athanassoula, E., pp. 177-186, Reidel, Dordrecht

Warren, M.S., Quinn, P.J., Salmon, J.K., Zurek, W.H., 1992. ApJ, 399, 405

Weiland at al, 1994. ApJ, 425, L81

Zhao, H.S., Rich, R.M., Spergel, D.N., 1994. ApJ, 000, 000 